\title{
DATA-DRIVEN TAXONOMY FOREST FOR FINE-GRAINED IMAGE CATEGORIZATION
}

\author{
Xiaomeng Wu, Minoru Mori, Kunio Kashino \\ NTT Communication Science Laboratories, Kanagawa, Japan 243-0198 \\ \{wu.xiaomeng,mori.minoru,kashino.kunio\}@lab.ntt.co.jp
}

\begin{abstract}
Fine-grained image categorization must handle huge crossclass ambiguities and a large number of classes. Inspired by the success of rigid hierarchical classification, we propose a new flexible hierarchical classification method, called a data-driven taxonomy forest. It constructs a multitude of taxonomies, each of which converts a complex multi-class problem to a more easily tractable path-finding problem. We demonstrate how a stochastic representation of local classification hypotheses incorporated in multiple taxonomies deals skillfully with error propagation and over-fitting. Various strategies for instance space decomposition are investigated from the viewpoint of taxonomy complexity. We comprehensively evaluate our data-driven taxonomy forest using Oxford Flower 102 and Oxford Pet benchmarks and show its superiority in effectiveness and generality to rigid hierarchical classification in fine-grained image categorization tasks.
\end{abstract}

Index Terms - Fine-grained image categorization, hierarchical classification, error propagation, bagging

\section{INTRODUCTION}

This paper addresses the problem of classifying images that belong to the same category at a basic level, e.g. species of plants and animals, which is known as fine-grained image categorization (FGIC). It is challenging because of the subtle variations between atomized classes [1,2]. We focus on solutions based on hierarchical classification (HC) inspired by their success in text categorization and function prediction $[3,4]$ that share a problem common to FGIC. Here, classes are organized into a taxonomy. For each test sample, the system predicts its first-level class, uses this class to narrow the choice of classes to be predicted at the second level, and so on, recursively, until the most specific prediction is made.

A disadvantage of traditional $\mathrm{HC}$ methods is that they rely on only one series of rigid decisions, as long as the length of the path from the root to the class at the leaf level, and are thus more likely to make mistakes. This is known as error propagation $[4,5]$ in the sense that an error at a certain level will propagate downward through the taxonomy. It is a crucial problem when the classifier over-fits the taxonomy and when the taxonomy is less consistent with the distribution of the

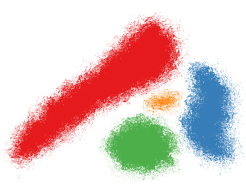

(a) Feature space

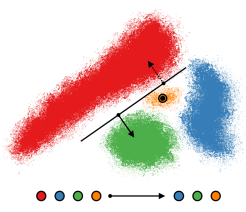

(d) Classifier at root

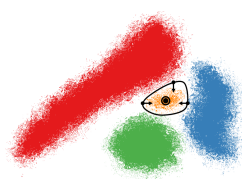

(b) Complex plane

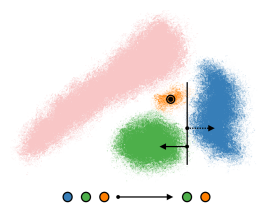

(e) Level 2

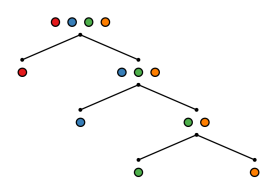

(c) Taxonomy

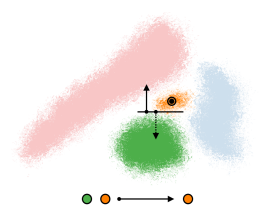

(f) Level 3

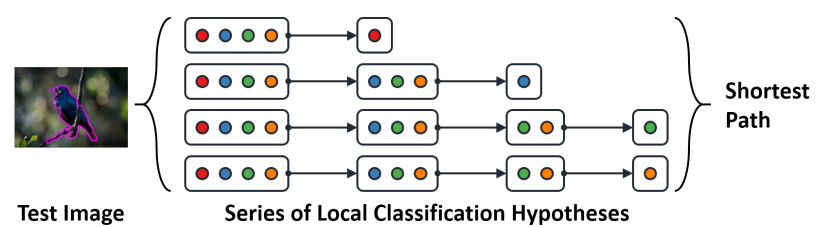

(g) Testing step of flexible hierarchical classification

Fig. 1: Flexible hierarchical classification. (a)-(f): difference between optimal hyperplanes of flat and local classifications. (g): each test image is passed through all inner nodes, resulting in multiple series of stochastic representations of local classification hypotheses; the leaf with the shortest path from the root is chosen as the winner.

classes in the instance space. Error propagation constitutes the main problem that we deal with in this paper.

Huang et al. [6] claimed that a perfect binary tree that minimizes complexity also performs better than an imbalanced binary tree in terms of accuracy. However, Deng et al. [7] showed that in learning a taxonomy, accuracy and complexity are conflicting goals in that accuracy optimization is accompanied by increased complexity. No evidence has been produced to confirm which theory is correct under identical conditions. In this paper, we answer this question by analyzing the classification loss of a flexible $\mathrm{HC}$ method and experimentally compare taxonomies with various complexities.

We propose a flexible HC method in Section 3. As shown in Fig. 1(g), each test image is passed through all inner nodes. The method maps the image to multiple series of stochastic representations of local classification hypotheses, rather than hard-mapping it to only one series of rigid decisions. It con- 
sequently localizes the image in the class space more flexibly and more precisely. We analyze the classification loss of our method, which motivates our choice among various strategies for instance space decomposition from the viewpoint of taxonomy complexity. To handle over-fitting, we develop a multitude of taxonomies and introduce variation among them by bootstrap splitting. In Section 4, we demonstrate the effectiveness and generality of our method by using various features with challenging public datasets.

\section{RELATED WORK}

FGIC has been explored for tasks such as the recognition of flowers [1, 2, 8,9], birds [1,9], fishes [6], and cats and dogs $[1,10]$. In co-segmentation methods $[1,9,10]$, shared appearance is exploited in images containing the same class of objects. Ito and Kubota [8] proposed heterogeneous cooccurrence features that performed well for recognizing flowers. Gao et al. [2] learned category-specific and shared dictionaries for better feature representation. These topics concern the field of FGIC but are not discussed any further because they are not related to the main objective of this research.

In $\mathrm{HC}$ methods, the taxonomy is either assigned by experts $[3,11]$ or learned by minimizing the generalization error of training data $[5,7]$. In the latter case, a common solution is to derive the taxonomy from the confusion matrix using divisive hierarchical clustering [5]. Huang et al. [6] claimed that a perfect binary tree that minimizes complexity also performs better than an imbalanced binary tree in terms of accuracy. Deng et al. [7] showed that accuracy and complexity are conflicting goals, and proposed a balancing term to make taxonomy complexity tunable.

Depending on the method used to represent local classification hypotheses [4], $\mathrm{HC}$ methods can be categorized into local classifier per node (LCN) $[5,7,11]$, local classifier per parent node (LCPN) [3], and big-bang types [12]. Tsochantaridis et al. [12] proposed a big-bang method based on a joint feature representation explicitly encoding the structure of the taxonomy. Binder et al. [11] proved that the structural support vector machine (SVM) facilitated by Tsochantaridis et al. [12] can be split into independent optimizations solved by onevs.-all SVMs assigned to each child node. The method boils down to LCN-based classification [5] whereby the training time can be reduced significantly. Instead of using one-vs.-all classifiers, Secker et al. [3] assigned a one-vs.-one classifier to each parent node, which is an example of LCPN-based classification, to halve the number of classifiers and to better model class ambiguities.

LCN, LCPN, and big-bang methods share a similar strategy during the testing phase, namely they rely on only one series of rigid decisions and are prone to error propagation. A blocking method [13] can avoid this problem to some extent, but does not support mandatory leaf node prediction. Tsai et al. [14] proposed utilizing both flat and local structural infor- mation throughout path decision-making to allow leaf node prediction with a smaller error-propagation risk.

We propose a flexible $\mathrm{HC}$ method in this paper, which differs from Bengio et al.'s [5] and Deng et al.'s [7] methods in terms of the representation of local classification hypotheses. We look at multiple series of stochastic decisions and the classifier relies on only one hard decision. Instead, the methods described above rely on only one series of hard decisions, as long as the length of the path from the root to the leaf. Our method also differs from random forests [15]. Our method decomposes the instance space by considering cross-class ambiguities, while Agrawal et al.'s method [15] decomposes the feature space randomly.

\section{DATA-DRIVEN TAXONOMY FOREST}

\subsection{Preliminaries}

Let $X$ be the image space and let $Y$ be a set of class labels. We assume that instances are pairs $(x, y)$ drawn according to a distribution over $X \times Y$. The taxonomy of classes $T=(V, E)$ is defined in the form of a full and directed binary tree, with a root $\perp$ and a parent relationship $\pi$ where $\pi(v)$ is the parent of node $v \in V \backslash\{\perp\}$. $E$ denotes the set of edges with a parentto-child orientation. Each class label $y \in Y$ constitutes a leaf node and $Y \subset V$. An inner node $v \in V \backslash Y$ is in the form of a set of classes. The sibling of each node $v \in V \backslash\{\perp\}$ is defined as $\mathfrak{S}(v)$ satisfying $v \cap \mathfrak{S}(v)=\varnothing$ and $\pi(v)=\pi(\mathfrak{S}(v))$. For each class $y \in Y$ we define the set of its ancestors $\mathfrak{P}(y)$ as

$$
\begin{aligned}
& \mathfrak{P}(y)=\left\{v_{1}^{y}, \cdots, v_{k_{y}}^{y}:\right. \\
& \left.v_{1}^{y}=y \wedge \forall l \in\left\{2, \cdots, k_{y}\right\}, v_{l}^{y}=\pi\left(v_{l-1}^{y}\right)\right\} .
\end{aligned}
$$

We present our data-driven taxonomy (DDT) in Sections 3.2 and 3.3 and extend it to a DDT forest (DDTF) in Section 3.4.

\subsection{Stochastic Hierarchical Classification}

We build a local classifier at each inner node. For classification, we consider a top-down classifier that makes flexible decisions at each inner node, instead of selecting the best class at each level and proceeding iteratively down the taxonomy.

We consider local classification hypotheses with two mapping functions: $\Phi: X \mapsto \mathbb{H}$, which encodes an image $x$ as a vectorial representation $\Phi(x)$, and $f: X \times V \mapsto[0,1]$, which encodes an instance $(x, v)$ as a flexible decision of the local classifier at $v$. These functions can be defined via deep learning, e.g. by using a convolutional neural network (CNN) [16]. However, the sophisticated structure of CNN makes overfitting a significant problem [16], especially when we only have a small number, e.g. 10 to 20 , of training instances per class. Therefore, we consider two shallow representations, namely locally-constrained linear coding (LLC) and a Fisher vector, to define $\Phi$ and pick LLC because the sparsity favored by LLC significantly reduces the memory required. 
Each node $v \in V \backslash\{\perp\}$ is tagged with a binary random variable $\mathbf{1}_{y \in v}$ that equals one if $y \in v$ and zero if $y \in \mathfrak{S}(v) . y$ is the label of a given image $x . \mathbf{1}_{y \in v}$ is distributed according to a conditional probability function $P\left(\mathbf{1}_{y \in v} \mid \mathbf{1}_{y \in \pi(v)}, x\right)$. The mapping function $f$ described above can thus be understood as a likelihood function $X \times V \mapsto P$. Inspired by the success of kernel methods in vision tasks, we consider kernel-based hypotheses $\hat{f}$ :

$$
\hat{f}(x, v)=\sum_{i=1}^{n} \alpha_{i} \mathbf{1}_{\hat{y}_{i} \in v} K\left(\Phi(x), \Phi\left(\hat{x}_{i}\right)\right)+b,
$$

where $x$ is the given image, $\alpha_{i}$ is the coefficient for the $i$-th training instance $\left(\hat{x}_{i}, \hat{y}_{i}\right)$, and $K: X \times X \mapsto \mathbb{R}$ is a positive definite and symmetric kernel. Equation 2 represents a nonprobabilistic binary SVM separating $v$ and $\mathfrak{S}(v)$. It gives approximates of pairwise class probabilities $f(x, v)$ :

$$
f(x, v)=\frac{1}{1+\exp (A \hat{f}(x, v)+B)},
$$

where $A$ and $B$ are estimated by minimizing the negative $\log$ likelihood of training instances.

Let $P(y \mid x)$ be the probabilistic model for a final classifier, i.e. a model over a class label $y$ conditional on a test image $x$. Given Eq. 1 denoting the series of all ancestors of a leaf $y$ heading from the root, we know that $\mathfrak{P}(y)$ is unique with respect to $y$ since each node in a binary tree has at most one parent. Therefore, we have

$$
\begin{aligned}
P(y \mid x) & =\prod_{v \in \mathfrak{P}(y) \backslash\{\perp\}} P\left(\mathbf{1}_{y \in v} \mid \mathbf{1}_{y \in \pi(v)}, x\right) \\
& =\prod_{v \in \mathfrak{P}(y) \backslash\{\perp\}} f(x, v) .
\end{aligned}
$$

We treat the taxonomy as a directed, weighted graph, and the classifier finds the shortest path between the root $\perp$ and the set of leaf nodes $Y$. The decision rule thus consists in choosing the class with the highest class posterior probability: $y_{m}(x)=\arg \max _{y \in Y} P(y \mid x)$. Our method relies on only a single rigid decision and so is less sensitive to error propagation. In contrast, rigid $\mathrm{HC}$ methods rely on a large number of risky rigid decisions of as many as $|\mathfrak{P}(y)|-1$.

\subsection{Instance Space Decomposition}

Here, we explain the process of taxonomy learning based on training instances $(x, y)$. We define an associated function

$$
g:(x, y) \in X \times Y \mapsto \frac{\max _{y^{\prime} \in Y \backslash\{y\}} P\left(y^{\prime} \mid x\right)}{P(y \mid x)}
$$

such that the sign of $1-g(x, y)$ defines a decision rule that amounts to: assign $x$ to $y$ if and only if $g(x, y)<1$. The learning problem is to find a taxonomy $T$ such that the classification loss $\mathcal{E}(g)=\mathbb{E}_{(x, y)}[P(g(x, y) \geq 1)]$ is minimal. Here, we define $\mathbb{E}$ as the geometric mean, and approximate $\mathcal{E}(g)$ by looking directly at $g(x, y)$. We are then left with the problem of upper bounding $\mathbb{E}_{(x, y)}[g(x, y)]$.

Let $S$ be a set of training instances drawn i.i.d. over $X \times$ $Y$ so that each class has the same number of instances, and let $\varphi_{+}=\mathbb{E}_{v \in \mathfrak{P}(y) \backslash\{\perp\}}[f(x, v)]$. Let $h_{y}=|\mathfrak{P}(y)|-1$ be the length traversed from the root to $y$, and let $\eta=\sum_{y \in Y} h_{y} /|Y|$ be its arithmetic mean. Then, the classification loss of our method described in Section 3.2 is upper bounded for all $T$ by:

$$
\mathbb{E}_{(x, y)}[g(x, y)] \leq \frac{1}{2 \varphi_{+}^{\eta}},
$$

the proof of which is given in the supplementary material. As discussed in this material, the classification loss is controlled in Eq. 6 by a trade-off between the stochastic margin $\varphi_{+}$and the complexity $\eta . \eta$ in a full binary tree falls within:

$$
\log _{2}|Y| \leq \eta \leq \frac{|Y|+1}{2}-\frac{1}{|Y|} .
$$

Here, the minimum can be reached by a perfect binary tree. The maximum corresponds to the most imbalanced binary tree, at each level of which either $v$ or $\mathfrak{S}(v)$ is identified with a leaf node. When $S$ is well balanced, i.e. the classes to be predicted have a distribution in the right equilibrium, both $\varphi_{+}$ and $\eta$ favor a perfect binary tree, which is in accordance with the conclusion drawn by Huang et al. [6]. This insight indicates that accuracy and complexity are not always conflicting, and provides a counter-example of the conclusion reached by Deng et al. [7]. However, the above case is seldom viable in large-scale FGIC, where $S$ is usually highly imbalanced. In such cases, we can build a perfect binary tree to reduce $\eta$, at the expense of lowering $\varphi_{+}$; otherwise, we maximize $\varphi_{+}$and expect that $\varphi_{+}$will be sufficient to overcome the effect of $\eta$.

Incorporating both $\varphi_{+}$and $\eta$ in taxonomy learning increases the degree of freedom. It makes the method more sensitive to over-fitting when we have 102 classes with only 10 to 20 training instances per class [17]. Therefore, we follow Bengio et al.'s formulation [5] and derive the taxonomy from the confusion matrix to maximize $\varphi_{+}$. We build the confusion matrix and define the cross-class ambiguity function by using the pairwise class probabilities in Eq. 3, instead of predicting only a label as in Bengio et al.'s method [5]. It is preferable to deliver easily separable classes in top positions in the taxonomy, as shown in Fig. 1, to minimize the effect of error propagation. Therefore, $\varphi_{+}$should be lower bounded by the margins at parents of leaf nodes. That is, it is the margins near the leaves, rather than those near the root, that determine the upper bound of Eq. 6. We thus employ agglomerative rather than divisive hierarchical clustering [5] on the confusion matrix to maximize the margins near the leaves. We use the single-linkage criterion because it ensures that $v$ and $\mathfrak{P}(v)$ at each node $v \in V \backslash\{\perp\}$ have the largest interval in the instance space. In Section 4.2, we show how our method outperforms both the perfect binary tree [6] and the most imbalanced binary tree. 


\subsection{Bootstrap Splitting}

The training set $S$ is split into sub-training and validation sets for building the confusion matrix and learning the taxonomy. The taxonomy and local classifiers may over-fit this one-time split when we have a small number of sub-training instances vs. large numbers of classes and feature dimensions. In this paper, we propose a novel combination of a forest-structured model and our flexible $\mathrm{HC}$ method to address this issue.

Given a training set $S$ of size $m$, let $S_{i}$ denote the set of instances associated with a label $y_{i}$ with $i \in\{1, \cdots,|Y|\}$ and let $m_{i}$ denote its size. For each $y_{i}$, we generate $N$ splits resulting in $N$ pairs of sub-training and validation sets denoted by $\mathcal{T}_{i}$ and $\mathcal{V}_{i}$, respectively. Since both $\left|\mathcal{T}_{i}\right|$ and $\left|\mathcal{V}_{i}\right|$ should be sufficiently large to avoid over-fitting, the best choice is to set $\left|\mathcal{T}_{i}\right|=\left|\mathcal{V}_{i}\right|=m_{i} / 2$ resulting in a pair of isometric sets. Splitting is realized by sampling $m_{i} / 2$ sub-training instances from $S_{i}$ uniformly and without replacement, with the remainder being validation instances.

Combining $\mathcal{T}_{i}$ and $\mathcal{V}_{i}$ for all classes gives us $N$ splits of multi-class sub-training and validation sets denoted by $\mathcal{T}^{j}$ and $\mathcal{V}^{j}$, respectively, with $j \in\{1, \cdots, N\} . N$ taxonomies are fitted using the $N$ splits, and local classifiers are trained independently. Given a leaf $y$ and a test image $x$, the unified class membership is defined by averaging the class posterior probability in Eq. 4 computed from the $N$ taxonomies:

$$
P(y \mid x)=\frac{1}{N} \sum_{j=1}^{N} P\left(y \mid x, T_{j}\right),
$$

where $T_{j}$ denotes the $j$-th taxonomy. The winner class $y_{m}(x)$ is chosen such that $y_{m}(x)=\arg \max _{y \in Y} P(y \mid x)$. Increasing $N$ decreases the variance of the forest, and so the training and testing errors tend to level off after a certain number of taxonomies have been fitted.

\section{EXPERIMENTS}

\subsection{Experimental Setting}

We report results obtained with our methods, namely datadriven taxonomy (DDT) and data-driven taxonomy forest (DDTF), for two FGIC benchmarks: Oxford Flower 102 [17] and Oxford Pet [10]. The former consists of 102 flower species and 8,189 images, with each category containing between 40 and more than 200 images. Twenty training images are predefined for each category while the remainder are used for testing. We start with a five-iteration graph cut initialized by a rectangle that constitutes $50 \%$ of the image size. The cut gives us a same segmentation mask for all compared methods. Oxford Pet is a challenging dataset containing 37 cat and dog species and 7,349 images. It has 100 training images for each category and provides rough foreground masks.

Color and local feature descriptors were extracted solely from the segmentation mask. Here, we considered two types
Table 1: Accuracies of DDTs with various complexities. Testing time (ms.) is averaged per feature and per image.

\begin{tabular}{lcccr}
\hline Oxford Flower 102 & RGB & D-SIFT & SIFT & Time \\
\hline Perfect Binary Tree [6] & 37.9 & 49.0 & 35.4 & 1.6 \\
Most Biased Binary Tree & 48.3 & 64.6 & 50.4 & 8.4 \\
Our Method & $\mathbf{4 9 . 0}$ & $\mathbf{6 8 . 1}$ & $\mathbf{5 2 . 5}$ & 7.8 \\
\hline \hline Oxford Pet & RGB & D-SIFT & SIFT & Time \\
\hline Perfect Binary Tree [6] & 19.4 & 44.3 & 29.7 & 1.8 \\
Most Biased Binary Tree & 20.0 & 50.4 & 32.2 & 5.6 \\
Our Method & $\mathbf{2 1 . 6}$ & $\mathbf{5 3 . 3}$ & $\mathbf{3 3 . 3}$ & 4.8 \\
\hline
\end{tabular}

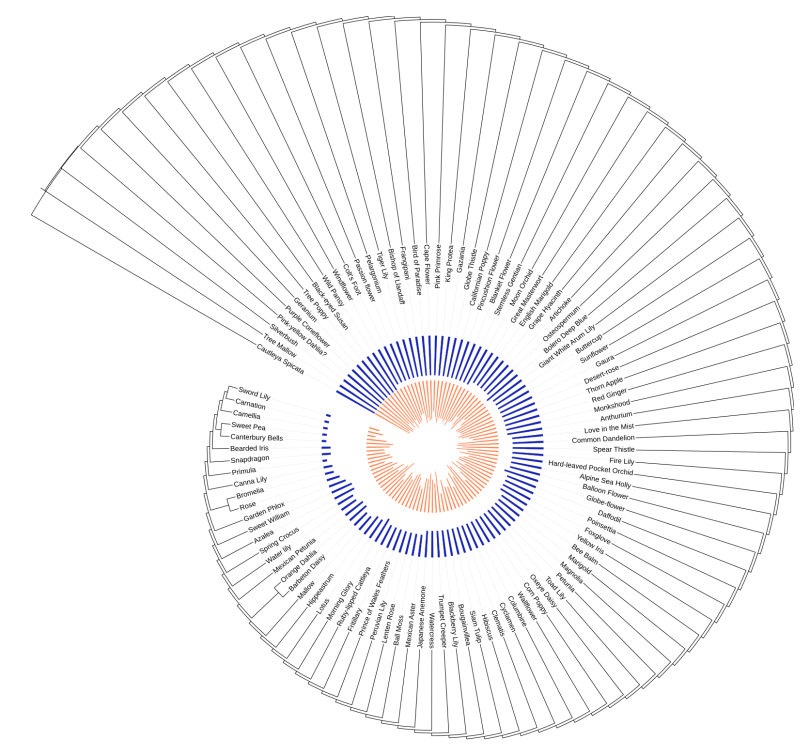

Fig. 2: DDT learned from Oxford Flower 102 by using our method. The root is at the upper left. The accuracies of the flat SVM for each category are shown in blue and our accuracies are shown in orange.

of local features. D-SIFT denotes the scale-invariant feature transform [18] extracted by dense sampling with one $32 \times 32$ scale; SIFT denotes the same feature extracted by feature detectors. LLC [19] with a three-level spatial pyramid model was used for feature encoding. CNN [16] may also be used here if sufficient data are available. The vocabulary sizes were 1,000 for color and 8,000 for D-SIFT and SIFT. Local classifiers were built via a linear SVM.

\subsection{Taxonomy Complexity}

Table 1 shows the accuracies for DDTs with various complexities. The most imbalanced binary tree was built by using recursive leave-one-out splits. Each split moves one level down the taxonomy. For each split, the most easily separable class $y$ is picked out, and the remainder constitutes its sibling $\mathfrak{S}(y)$.

From Table 1, we can see that the perfect binary tree [6] significantly underperformed the others. High-quality discrimination based on Huang et al.'s method [6] depends heavily on the assumption that the classes have a well-balanced distribution. This assumption seldom holds when we have 
Table 2: Accuracies of hierarchical classification methods. Testing time (ms.) is averaged per feature and per image.

\begin{tabular}{lcccr}
\hline Oxford Flower 102 & RGB & D-SIFT & SIFT & Time \\
\hline Flat SVM & 47.7 & 63.8 & 53.2 & 0.9 \\
\hline Secker et al. [3] & 16.9 & 8.4 & 14.4 & 3.1 \\
Label Trees [5, 11] & 38.6 & 59.9 & 43.2 & 13.6 \\
CLHC [14] & 47.9 & 63.2 & 52.3 & 71.0 \\
\hline DDT & 49.0 & 68.1 & 55.7 & 7.8 \\
DDTF (6 Trees) & 50.9 & 71.0 & 57.5 & 46.9 \\
DDTF (11 Trees) & $\mathbf{5 1 . 0}$ & $\mathbf{7 1 . 1}$ & $\mathbf{5 7 . 9}$ & 85.9 \\
\hline \hline Oxford Pet & RGB & D-SIFT & SIFT & Time \\
\hline Flat SVM & 21.0 & 51.2 & 33.4 & 0.7 \\
\hline Secker et al. [3] & 7.2 & 23.7 & 9.7 & 1.7 \\
Label Trees [5,11] & 12.8 & 46.7 & 25.9 & 9.0 \\
CLHC [14] & 21.2 & 50.8 & 33.3 & 6.7 \\
\hline DDT & 21.6 & 53.3 & 33.3 & 4.8 \\
DDTF (6 Trees) & $\mathbf{2 2 . 1}$ & 54.2 & 35.9 & 28.8 \\
DDTF (11 Trees) & 21.9 & $\mathbf{5 4 . 4}$ & $\mathbf{3 6 . 0}$ & 52.8 \\
\hline
\end{tabular}

a large number of categories, e.g. 102 species in the flower dataset. Meanwhile, the performance of the most imbalanced binary tree is comparable to that of our method. This indicates that the training set is highly imbalanced for both datasets, and the binary tree that optimizes the stochastic margin $\varphi_{+}$ should have a similar structure to the most imbalanced binary tree. The binary tree learned using our method is shown in Fig. 2, which corroborates the above discussion. As a future work, we shall find a balanced dataset and verify whether a balanced or an imbalanced tree is better in this case.

\subsection{Evaluation and Discussion}

We implemented several advanced HC methods and compare them in Table 2. Secker et al.'s method [3] is LPCN-based and performs in the same way as DDT except that it makes rigid decisions at each inner node. Label trees $[5,11]$ are LCNbased, and a one-vs.-all classifier is trained for each child instead of a binary classifier for each parent. Cross-level hierarchical classification (CLHC) [14] is a rigid HC method designed for handling error propagation. It chooses the best child for each parent recursively by first finding the best leaf from a semi-local multi-class classifier and then returning to the current ancestor of the leaf.

Most traditional HC methods in Table 2 behaved much worse than the flat SVM. They are generally designed with a taxonomy of only several levels in mind, while in our experiments we are dealing with many classes leading to a taxonomy of tens of levels. Rigid HC based on such a deep taxonomy is sensitive to error propagation when the categories derive subtle cross-class variations. Label trees $[5,11]$ exhibited greater robustness as regards error propagation at the expense of lower efficiency because they impose a much larger-scale

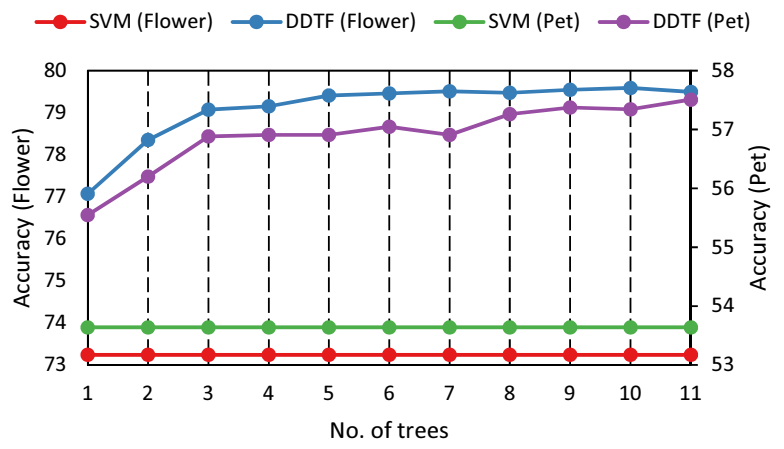

Fig. 3: Accuracy (\%) vs. number of trees in DDTF.

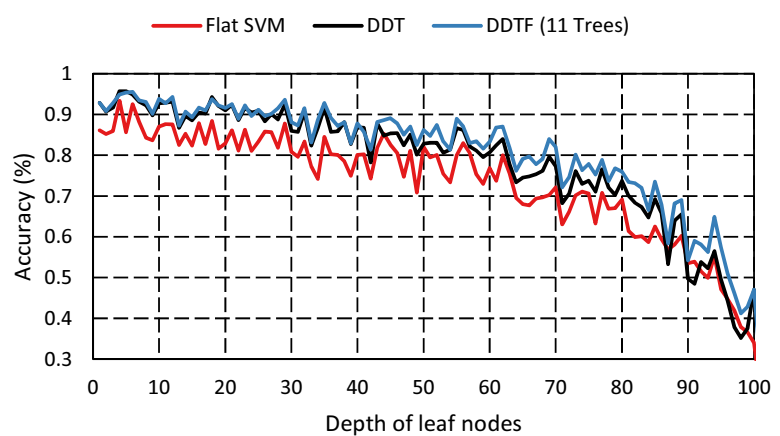

Fig. 4: Accuracy vs. depth of leaves in DDTF. For each depth value, all leaves with the same depth as this value in all 11 trees are collected, over which the accuracies of all three methods are averaged.

one-vs.-all classification on each node. CLHC [14] outperformed the above methods although it did not match the performance of SVM and DDT. Note that D-SIFT outperformed SIFT because transformation invariance pales by comparison with the integrity of feature extraction when the objects to be categorized are deformable.

DDT was greatly superior to all HC methods in terms of accuracy, thanks to the stochastic representation of local classification hypotheses. It also showed a higher efficiency than CLHC [14]. DDTF required more testing time than the flat SVM, but the actual speed was acceptable, namely less than $0.1 \mathrm{sec}$. per image for an 11-tree DDTF. As a reference, extracting LLC for SIFT took around three sec. per image. Nevertheless, we shall revisit our hierarchical classification flow for examination of the scope for further acceleration.

\subsection{Overall Accuracy Comparison}

In this experiment, we concatenate the sparse representations of RGB, D-SIFT, and SIFT together with corresponding weights $\{0.25,0.375,0.375\}$. The relation between accuracies and numbers of trees is shown in Fig. 3. Accuracies leveled off after several trees were fitted. Unlike the random forest [15], which typically requires hundreds of trees, DDTF stabilizes the performance with incomparably fewer DDTs.

We explored the relation between accuracies and depths of leaf nodes. $\mathfrak{P}(v)$ is measured as the depth of each leaf $y$. For each depth value, all leaf nodes with the same depth as 
Table 3: Reported accuracies in literature.

\begin{tabular}{lcr}
\hline Methods & Oxford Flower 102 & Oxford Pet \\
\hline Ito and Kubota [8] & 74.8 & - \\
Chai et al. [9] & 79.4 & - \\
Parkhi et al. [10] & - & 54.1 \\
Angelova and Zhu [1] & $\mathbf{8 0 . 7}$ & 54.3 \\
DDT & 77.1 & 55.5 \\
DDTF & 79.5 & $\mathbf{5 7 . 5}$ \\
\hline
\end{tabular}

this value in all 11 trees are collected, over which the accuracies of SVM, DDT, and DDTF are averaged. From Fig. 4, we can observe a smooth drop in the accuracy of SVM from the root to deep nodes, which corroborates the discussion in Section 3.3 that classes in top positions in the taxonomy are more easily separable than those near the leaves. DDT outperformed SVM but behaved unsteadily at deep nodes due to over-fitting. In contrast, although DDTF showed no improvement over DDT for classes near the root, it outperformed SVM more consistently even on a deep level.

We compare our methods with other methods in Table 3. DDTF outperforms all methods except with Oxford Flower 102, where it is outperformed by Angelova and Zhu's method [1]. The latter benefits from a more accurate segmentation than our method. For Oxford Flower 102, our segmentation method achieved a mean Jaccard coefficient of $89.3 \%$. We believe that our DDTF would outperform Angelova and Zhu's method [1] given the same segmentation.

\section{CONCLUSION}

We have proposed a flexible HC method for FGIC. In our experiments, our method significantly outperformed traditional HC methods and achieved state-of-the-art performance on two recent datasets. Our method allows elastic inference and introduces forest-structured $\mathrm{HC}$, which greatly mitigates error propagation and over-fitting. The classification loss of our method showed that overall accuracy, local classification margin, and complexity are not always conflicting. Their relation in fact depends on the class distribution in the instance space. We have not yet tested our method in multiple kernel learning scenarios. We shall deal with this issue in the future. We also intend to conduct experiments on larger datasets, e.g. ImageNet [20]. Such datasets provide large quantities of data, enabling us to use a CNN [16] for local classification. These topics constitute our future work.

\section{REFERENCES}

[1] A. Angelova and S. Zhu, "Efficient object detection and segmentation for fine-grained recognition," in CVPR, 2013, pp. 811-818.

[2] S. Gao, I. W.-H. Tsang, and Y. Ma, "Learning category-specific dictionary and shared dictionary for fine-grained image cate- gorization," IEEE Transactions on Image Processing, vol. 23, no. 2, pp. 623-634, 2014.

[3] A. Secker, M. N. Davies, A. A. Freitas, E. Clark, J. Timmis, and D. R. Flower, "Hierarchical classification of G-proteincoupled receptors with data-driven selection of attributes and classifiers," IJDMB, vol. 4, no. 2, pp. 191-210, 2010.

[4] C. N. Silla Jr. and A. A. Freitas, "A survey of hierarchical classification across different application domains," Data Min. Knowl. Discov., vol. 22, no. 1-2, pp. 31-72, 2011.

[5] S. Bengio, J. Weston, and D. Grangier, "Label embedding trees for large multi-class tasks," in NIPS, 2010, pp. 163-171.

[6] P. X. Huang, B. J. Boom, and R. B. Fisher, "Underwater live fish recognition using a balance-guaranteed optimized tree," in ACCV (1), 2012, pp. 422-433.

[7] J. Deng, S. Satheesh, A. C. Berg, and F. Li, "Fast and balanced: Efficient label tree learning for large scale object recognition," in NIPS, 2011, pp. 567-575.

[8] S. Ito and S. Kubota, "Object classification using heterogeneous co-occurrence features," in $\operatorname{ECCV}$ (2), 2010, pp. 209222.

[9] Y. Chai, V. S. Lempitsky, and A. Zisserman, "BiCoS: A bi-level co-segmentation method for image classification," in ICCV, 2011, pp. 2579-2586.

[10] O. M. Parkhi, A. Vedaldi, A. Zisserman, and C. V. Jawahar, "Cats and dogs," in CVPR, 2012, pp. 3498-3505.

[11] A. Binder, K.-R. Müller, and M. Kawanabe, "On taxonomies for multi-class image categorization," International Journal of Computer Vision, vol. 99, no. 3, pp. 281-301, 2012.

[12] I. Tsochantaridis, T. Joachims, T. Hofmann, and Y. Altun, "Large margin methods for structured and interdependent output variables," Journal of Machine Learning Research, vol. 6, pp. 1453-1484, 2005.

[13] A. Sun, E.-P. Lim, W. K. Ng, and J. Srivastava, "Blocking reduction strategies in hierarchical text classification," IEEE Trans. Knowl. Data Eng., vol. 16, no. 10, pp. 1305-1308, 2004.

[14] M.-H. Tsai, S.-F. Tsai, and T. S. Huang, "Hierarchical image feature extraction and classification," in ACM Multimedia, 2010, pp. 1007-1010.

[15] R. Agrawal, A. Gupta, Y. Prabhu, and M. Varma, "Multi-label learning with millions of labels: recommending advertiser bid phrases for web pages," in $W W W, 2013$, pp. 13-24.

[16] A. Krizhevsky, I. Sutskever, and G. E. Hinton, "ImageNet classification with deep convolutional neural networks," in NIPS, 2012, pp. 1106-1114.

[17] M.-E. Nilsback and A. Zisserman, "Automated flower classification over a large number of classes," in ICVGIP, 2008, pp. 722-729.

[18] D. G. Lowe, "Distinctive image features from scale-invariant keypoints," International Journal of Computer Vision, vol. 60, no. 2, pp. 91-110, 2004.

[19] J. Wang, J. Yang, K. Yu, F. Lv, T. S. Huang, and Y. Gong, "Locality-constrained Linear Coding for image classification," in CVPR, 2010, pp. 3360-3367.

[20] O. Russakovsky et al., "ImageNet large scale visual recognition challenge," in arXiv:1409.0575, 2014. 


\title{
DATA-DRIVEN TAXONOMY FOREST FOR FINE-GRAINED IMAGE CATEGORIZATION
}

\author{
Xiaomeng Wu, Minoru Mori, Kunio Kashino \\ NTT Communication Science Laboratories, Kanagawa, Japan 243-0198 \\ \{wu.xiaomeng,mori.minoru,kashino.kunio\}@lab.ntt.co.jp
}

\section{A. UPPER BOUND OF CLASSIFICATION LOSS}

In this appendix, we briefly analyze the classification loss of our method described in Section 3.3. Let us first recall Eq. 5:

$$
g:(x, y) \in X \times Y \mapsto \frac{\max _{y^{\prime} \in Y \backslash\{y\}} P\left(y^{\prime} \mid x\right)}{P(y \mid x)} .
$$

which gives rise to a decision rule that amounts to: assign $x$ to $y$ if and only if $g(x, y)<1$. The classification loss is thus $\mathcal{E}(g)=\mathbb{E}_{(x, y)}[P(g(x, y) \geq 1)]$ with $\mathbb{E}$ being the geometric mean. We approximate $\sup \mathcal{E}(g)$ by looking directly at $g$ and upper bounding $\mathbb{E}_{(x, y)}[g(x, y)]$.

Let $y^{*}=\arg \max _{y^{\prime} \in Y \backslash\{y\}} P\left(y^{\prime} \mid x\right)$ denote the class that is the most confusing with $y$ given $x$. By combining Eq. 4 and Eq. A, we have

$$
\begin{aligned}
g(x, y) & =\frac{\prod_{v^{*} \in \mathfrak{P}\left(y^{*}\right) \backslash\{\perp\}} f\left(x, v^{*}\right)}{\prod_{v \in \mathfrak{P}(y) \backslash\{\perp\}} f(x, v)} \\
& =\frac{\prod_{v^{*} \in \mathfrak{P}\left(y^{*}\right) \backslash \mathfrak{P}(y)} f\left(x, v^{*}\right)}{\prod_{v \in \mathfrak{P}(y) \backslash \mathfrak{P}\left(y^{*}\right)} f(x, v)}
\end{aligned}
$$

since $f(x, v)=f\left(x, v^{*}\right)$ for all $v \in \mathfrak{P}(y) \cap \mathfrak{P}\left(y^{*}\right)$. Let $\varphi_{+}$ and $\varphi_{-}$denote the geometric means of $f(x, v)$ and $f\left(x, v^{*}\right)$, respectively. Given $h_{+}=\left|\mathfrak{P}(y) \backslash \mathfrak{P}\left(y^{*}\right)\right|$ and $h_{-}=\mid \mathfrak{P}\left(y^{*}\right) \backslash$ $\mathfrak{P}(y) \mid$, we have

$$
g(x, y)=\frac{\varphi_{-}^{h_{-}}}{\varphi_{+}^{h_{+}}}
$$

It is understood that $f\left(x, v^{*}\right)$ at each $v^{*} \in \mathfrak{P}\left(y^{*}\right) \backslash \mathfrak{P}(y)$ classifies $x$ into classes to which $x$ does not belong, because $y \notin v^{*}$ holds for all such $v^{*}$. Given a real number $\delta \in[0,1]$, $\delta$ and $1-\delta$ have an equal chance of being observed from $f\left(x, v^{*}\right)$, and so $f\left(x, v^{*}\right)$ has an unknown distribution that is symmetric with respect to its arithmetic mean 0.5 . We thus have $\varphi_{-} \leq 0.5$, according to the inequality of arithmetic and geometric means, such that

$$
g(x, y) \leq \frac{0.5^{h_{-}}}{\varphi_{+}^{h_{+}}}
$$

Given $h_{y}=|\mathfrak{P}(y)|-1$ denoting the length traversed from the root to $y$, we have $h_{+} \leq h_{y}$ and $h_{-} \geq 1$, and so

$$
g(x, y) \leq \frac{0.5^{h_{-}}}{\varphi_{+}^{h_{+}}} \leq \frac{0.5}{\varphi_{+}^{h_{y}}}=\frac{1}{2 \varphi_{+}^{h_{y}}}
$$

Let $S=\left\{\left(x_{i}, y_{i}\right)\right\}_{i=1}^{m}$ be a set of $m$ training instances drawn i.i.d. over $X \times Y$ so that each class has the same number $m /|Y|$ of instances. The upper bound of $\mathbb{E}_{(x, y)}[g(x, y)]$ is thus

$$
\begin{aligned}
\mathbb{E}_{(x, y)}[g(x, y)] & =\prod_{(x, y) \in S} g(x, y)^{1 / m} \\
& \leq \prod_{(x, y) \in S} \frac{1}{\left(2 \varphi_{+}^{h_{y}}\right)^{1 / m}} \\
& \leq \prod_{y \in Y} \frac{1}{\left(2 \varphi_{+}^{h_{y}}\right)^{1 /|Y|}} \\
& =\frac{1}{2 \varphi_{+}^{\eta}}
\end{aligned}
$$

where $\eta=\sum_{y \in Y} h_{y} /|Y|$ is the mean length of paths heading from the root $\perp$ to any of the leaf nodes $y \in Y$.

\section{B. TRADE-OFF BETWEEN $\varphi_{+}$AND $\eta$}

On one hand, Equation 6 is a monotonically-decreasing function of $\varphi_{+}$, i.e. maximizing $\varphi_{+}$lowers the upper bound and so improves the classification. On the other hand, since $\varphi_{+} \leq 1$, Equation 6 is increasing as regards $\eta$ and so minimizing $\eta$ improves the classification.

By the properties of binary trees, the minimum of $\eta$ is reached when the hierarchy is perfectly balanced and in this case $\eta=\log _{2}|Y|$. When the hierarchy is the most imbalanced, $\eta$ reaches its maximum and equals $\frac{|Y|+1}{2}-\frac{1}{Y}$. In a nutshell, $\eta$ depends only on the balance of the binary tree and does not depend on $\varphi_{+}$.

In contrast, $\varphi_{+}$has no direct relationship to the balance of the binary tree. It depends only on whether the balance of the binary tree coincides with the distribution of the classes in the instance space. When the classes to be predicted have a distribution in the right equilibrium, the maximum of $\varphi_{+}$ is reached only when the hierarchy is perfectly balanced. In this case, Equation 6 is optimized by $\varphi_{+}$and $\eta$ at the same time. However, when the distribution of the classes is highly imbalanced, the stochastic margin $\varphi_{+}$is to be small if we forcibly use a balanced binary tree. To increase $\varphi_{+}$, we can consider only an imbalanced binary tree, but that increases $\eta$. In this sense, we say that the classification loss is controlled by a trade-off between $\varphi_{+}$and $\eta$. 